\title{
CASE REPORT \\ Diffusion tensor imaging and tractography in brown-sequard syndrome
}

\author{
A Vedantam ${ }^{1}$, MB Jirjis ${ }^{2}$, BD Schmit ${ }^{2}$, MD Budde $^{1}$, JL Ulmer $^{3}$, MC Wang $^{1}$ and SN Kurpad ${ }^{1}$
}

Study design: Case report.

Objective: To demonstrate the utility of diffusion tensor imaging (DTI) and tractography in two patients with Brown-Sequard syndrome after penetrating cervical cord injury.

Setting: Milwaukee, WI, USA.

Methods: Two patients, who presented with features of Brown-Sequard syndrome after sustaining stab wounds to the neck, underwent DTI and tractography of the cervical cord within a week of the injury. DTI metrics were measured within the left and right hemicord around the level of injury. Diffusion tensor tractography (DTT) was performed to visualize the site of injury and injured fiber tracts.

Results: Axial fractional anisotropy (FA) maps at the site of injury showed unilateral damage to the cord structure, and FA was significantly reduced within the injured hemicord in both patients. Tractography allowed for visualization of the injured fiber tracts around the level of injury. Both DTI metrics and tractography showed an asymmetry that corresponded to the neurological deficits exhibited by the patients.

Conclusion: This report illustrates the utility of DTI and DTT in delineating regions of cord injury in two patients with traumatic Brown-Sequard syndrome. Our results indicate that DTI provides clinically relevant information that supplements conventional MR imaging for patients with acute $\mathrm{SCl}$.

Spinal Cord (2012) 50, 928-930; doi:10.1038/sc.2012.94; published online 9 October 2012

Keywords: diffusion tensor imaging; spinal cord injury; cervical spine; brown-Sequard syndrome; fractional anisotropy

\section{INTRODUCTION}

Diffusion tensor imaging (DTI) metrics within the spinal cord are sensitive to neural damage in patients with acute spinal cord injury (SCI). ${ }^{1}$ We report the use of DTI and tractography to characterize two cases of Brown-Sequard syndrome (BSS) following penetrating cervical cord injury. We sought to determine whether DTI metrics show anatomical asymmetry that matches clinical presentation.

\section{CASE REPORTS}

\section{Patient 1}

A 51-year old male presented with stab wounds to the neck following assault. On examination, he had decreased motor strength in the left upper and lower limbs (MRC grades: elbow—4/5, wrist-4/5, hip/knee/ ankle-0/5). On the right side, he had 5/5 motor strength throughout. Sensory examination revealed impaired pinprick sensation only on the right side. Light touch and proprioception were intact bilaterally. On the left, knee jerk was absent and ankle jerk was diminished. Plantar reflexes were flexor bilaterally.

\section{Patient 2}

A 32-year old male presented to the ER following a stab wound to the neck. Motor examination revealed weakness on the right side (maximum MRC grade: upper limb-3/5, lower limb-3/5). Sensory examination showed absent pinprick sensation in the left upper and lower extremities and diminished sensation to light touch in the right upper and lower limb. CSF leakage was noted from the wound, which was closed primarily with deep subcutaneous sutures.

\section{Imaging protocol}

Both patients underwent DTI using a 1.5T MR scanner within a week following injury (Patient 1-6 days, Patient 2-48h). A single-shot, echo planar pulse sequence was used with a twice-refocused spin-echo diffusion preparation. Axial diffusion-weighted images $(3 \mathrm{~mm}$ thick with a $0.5 \mathrm{~mm}$ gap between slices) were acquired along 15 distinct directions at a b-value of $600 \mathrm{~s} / \mathrm{mm}^{2}$ using a TR/TE of 5000/98.2 ms, matrix size of $128 \times 128$, and FOV of $19 \mathrm{~cm}^{2}$.

Images were processed using Analysis of Functional NeuroImages processing suite (AFNI, http://afni.nimh.nih.gov/afni/) and Matlab (MathWorks, Natick, MA, USA). Regions of interest within the whole cord, and right and left halves of the cord were drawn on axial fractional anisotropy (FA) maps by two investigators (AV and MBJ) independently. The average DTI indices are reported. Diffusion tensor tractography (DTT) was performed using DSI Studio (http://dsi-studio. labsolver.org). Tractography was performed using a Runge-Kutta algorithm to generate 1500 tracts using an angle threshold of $45^{\circ}$, FA threshold of 0.18 , step size of $0.1 \mathrm{~mm}$, and length constraint of $10 \mathrm{~mm}$.

${ }^{1}$ Department of Neurosurgery, Medical College of Wisconsin, Milwaukee, WI, USA; ${ }^{2}$ Department of Biomedical Engineering, Marquette University, Milwaukee, WI, USA and ${ }^{3}$ Department of Radiology, Medical College of Wisconsin, Milwaukee, WI, USA

Correspondence: Dr SN Kurpad, Department of Neurosurgery, Medical College of Wisconsin, Chief, Neurosurgical Service, Clement J Zablocki VA Medical Center, 9200W Wisconsin Avenue, Milwaukee, WI 53226, USA.

E-mail: skurpad@mcw.edu

Received 3 May 2012; revised 11 July 2012; accepted 15 July 2012; published online 9 October 2012 
Cervical cord segments showing intramedullary hyperintensity on sagittal T2W images were defined as the injury zone. FA maps and DTT within the injury zone are shown in Figures 1 and 2. DTI metrics within the injury zone are shown in Table 1 . There was an asymmetry in DTI metrics that corresponded with physical exam.

\section{DISCUSSION}

Brown-Sequard syndrome is characterized by ipsilateral motor weakness and hypoesthesia, with contralateral loss of pain and temperature sensation below the level of injury. ${ }^{2}$ However, the classical syndrome is rarely seen, and patients often present with only asymmetric paresis and hypalgesia on the less paretic side-termed 'Brown-Sequard plus syndrome.' In the present report, patient 2 presented with the classical syndrome, while patient 1 had features of the 'plus' syndrome. Although a number of aetiologies have been reported, ${ }^{4}$ penetrating SCI continues to be a common cause of Brown-Sequard syndrome. Stab wounds, in particular, predispose to this type of

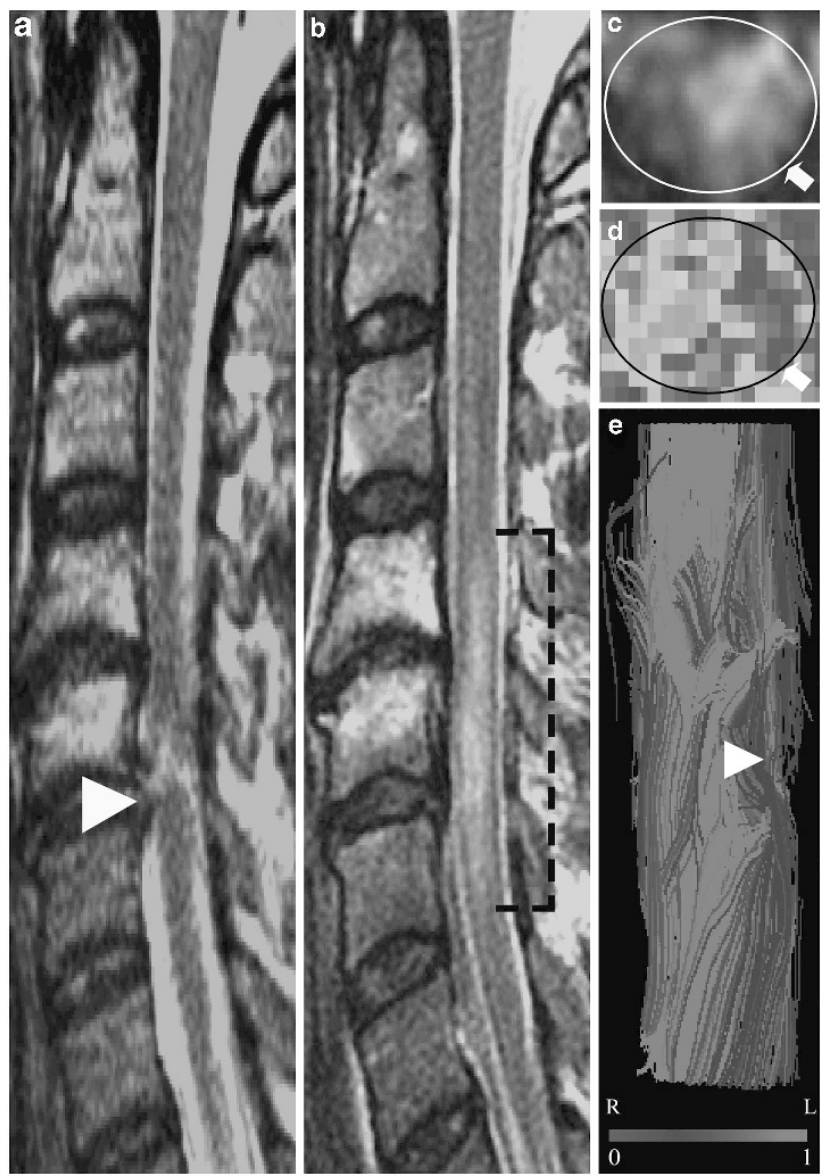

Figure 1 (a) T2W left parasagittal image of the cervical spine in Patient 1 showing the site of injury (arrow head); (b) Mid-sagittal T2W image showing intramedullary hyperintensity between C4-C6 (injury zone). The segments of the cord demarcated by the dotted line were selected for tractography; (c) Axial T2W image at the injury site showing ill-defined hyperintensity within the left hemicord. Block arrow shows the direction of injury; (d) Axial FA map at the injury site showing obliteration of the cord structure (solid line) particularly within the left hemicord (block arrow) (A-anterior, $\mathrm{P}$-posterior); (e) Anterior view of fiber tracts around the site of injury (arrow head) shows disrupted white matter tracts and low anisotropic fibers on the left half of the cord (color bar represents mean anisotropy) (R-right, L-left). A full color version of this figure is available at the Spinal Cord journal online. injury since the orientation of the spinous processes and transverse processes of the vertebrae creates a groove that allows the blade to unilaterally injure the cord. ${ }^{5}$ The prognosis in patients with Brown-Sequard syndrome is considered to be better than complete

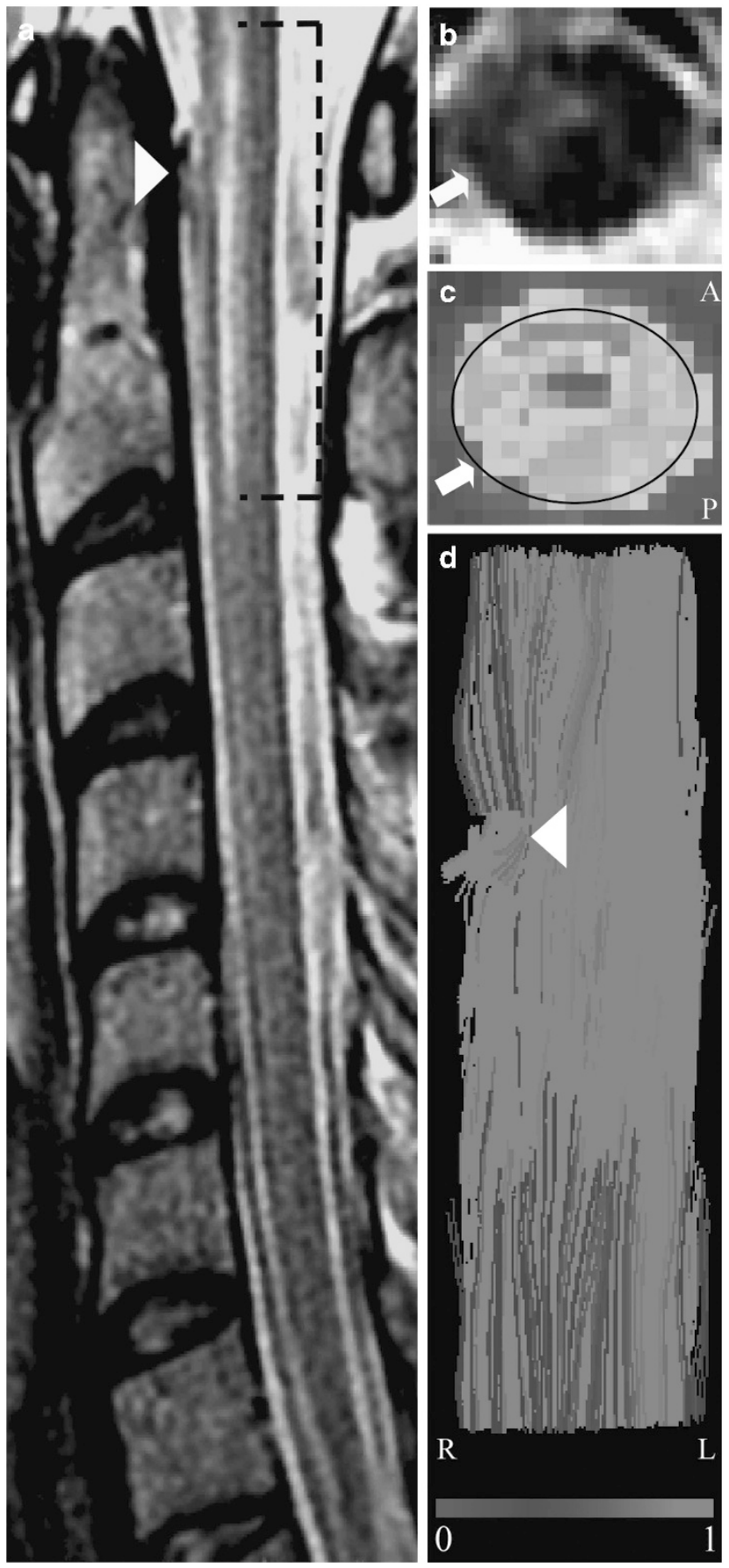

Figure 2 (a) Sagittal T2W cervical spine image in Patient 2 showing a linear hyperintensity between C1-C2 (injury zone). Arrow head shows the site of injury, and segments within the dotted line were selected for tractography; (b) T2W axial image of the cervical cord at the site of injury showing hyperintensity in the right half of the cord (block arrow); (c) Axial FA map showing the posterolateral site of injury (block arrow) and incomplete cord structure (intact cord structure depicted by solid line); (d) Anterior view of fiber tracts showing the site of injury on the right (arrow head) (R-right, L-left) (color bar represents mean anisotropy). A full color version of this figure is available at the Spinal Cord journal online. 
Table 1 DTI metrics at the injury zone in 2 patients with traumatic Brown-Sequard syndrome

\begin{tabular}{lllll}
\hline & Whole cord & Right & Left & P value \\
\hline $\begin{array}{l}\text { Patient 1 } \\
\text { Injury zone (C4-C6) }\end{array}$ & & & & \\
$\quad$ FA & $0.48(0.02)$ & $0.53(0.08)$ & $0.41(0.08)$ & $0.004^{*}$ \\
MD $\left(\times 10^{-3} \mathrm{~mm}^{2} \mathrm{~s}^{-1}\right)$ & $1.32(0.05)$ & $1.2(0.2)$ & $1.51(0.1)$ & $0.003^{*}$ \\
& & & & \\
Patient 2 & & & & \\
$\quad$ Injury zone (C1-C2) & & & & \\
$\quad$ FA & $0.47(0.01)$ & $0.45(0.04)$ & $0.49(0.04)$ & $0.009 *$ \\
MD $\left(\times 10^{-3} \mathrm{~mm}^{2} \mathrm{~s}^{-1}\right)$ & $0.91(0.02)$ & $0.91(0.1)$ & $0.89(0.1)$ & 0.54 \\
\hline
\end{tabular}

FA, fractional anisotropy; MD, mean diffusivity. Indices of the injured hemicord are in bold. ${ }^{*} P<0.01$.

SCI, while patients with Brown-Sequard plus syndromes tend to have more favorable outcomes as compared to those with the classical syndrome. ${ }^{3}$

To the best of our knowledge, only one previous study has documented the role of DTI and DTT in patients with penetrating SCI. ${ }^{6}$ In that study, DTI was performed in a single patient with BSS 2 months after a thoracic stab injury. Similar to their results, we found lower FA values and higher MD values at the injury zone. Importantly, FA was significantly reduced within the injured hemicord while DTT showed unilateral tract disruption and regions of low anisotropy. As compared to conventional MR imaging, both DTI and DTT provided superior visualization of the asymmetrically injured cervical cord. Additionally, the information provided by DTI and DTT was consistent with the neurological deficits, thereby proving to be clinically relevant.

Previous clinical reports have documented decreased FA and MD in the cervical spinal cord within $24 \mathrm{~h}$ post-SCI. ${ }^{1,7}$ In the present report, both patients showed an increase in MD that may be attributed to progressive lesion growth, as described previously in animal SCI models. ${ }^{8}$ This suggests a possible trend in DTI metrics associated with an evolving spinal cord lesion. However, a larger study population is necessary to determine variations in DTI metrics over time after acute SCI. Such a study would also require that patients undergo DTI at consistent time intervals after the injury.

Presently, spinal cord DTI is performed in a modest number of major medical centers in the United States. At our center, we are able to perform DTI using a standard clinical MR scanner with a dedicated spine coil. Although no additional equipment is required, specific DTI sequences need to be setup and refined to acquire reliable DTI images. ${ }^{9}$ DTI images, then, require post-processing using either commercially available software or in-house custom software. While spinal cord DTI is currently used as a research tool, it has the potential to significantly supplement conventional MR imaging in a clinical setting. The clinical implementation of spinal cord DTI, however, requires the development of standardized scan sequences as well as robust post-processing systems.

\section{CONCLUSION}

This report illustrates the utility of DTI and DTT in delineating regions of cord injury in two patients with traumatic BSS. Our results indicate that DTI provides clinically relevant information that supplements conventional MR imaging for patients with acute SCI.

\section{CONFLICT OF INTEREST}

The authors declare no conflict of interest.

\section{ACKNOWLEDGEMENTS}

VA Rehab R\&D grant \#1 I01 RX000113-01, Bryon Riesch Paralysis Foundation Endowment.

1 Shanmuganathan K, Gullapalli R, Zhuo J, Mirvis S. Diffusion tensor MR imaging in cervical spine trauma. AJNR Am J Neuroradiol 2008; 29: 655-659.

2 Aminoff MJ. Historical perspective Brown-Sequard and his work on the spinal cord. Spine 1996; 21: 133-140.

3 Roth EJ, Park T, Pang T, Yarkony GM, Lee MY. Traumatic cervical Brown-Sequard and Brown-Sequard-plus syndromes: the spectrum of presentations and outcomes. Paraplegia 1991; 29: 582-589.

4 Koehler PJ, Endtz LJ. The Brown-Sequard syndrome. True or false? Arch Neurol 1986; 43: 921-924.

5 Peacock WJ, Shrosbree RD, Key AG. A review of 450 stabwounds of the spinal cord S Afr Med J 1977; 51: 961-964.

6 Rajasekaran S, Kanna RM, Karunanithi R, Shetty AP. Diffusion tensor tractography demonstration of partially injured spinal cord tracts in a patient with posttraumatic Brown Sequard syndrome. J Magn Reson Imaging 2010; 32: 978-981.

7 Cheran S, Shanmuganathan K, Zhuo J, Mirvis SE, Aarabi B, Alexander MT et al. Correlation of MR diffusion tensor imaging parameters with ASIA motor scores in hemorrhagic and nonhemorrhagic acute spinal cord injury. J Neurotrauma 2011; 28 1881-1892.

8 Ellingson BM, Schmit BD, Kurpad SN. Lesion growth and degeneration patterns measured using diffusion tensor 9.4-T magnetic resonance imaging in rat spinal cord injury. J Neurosurg Spine 2010; 13: 181-192.

9 Maier SE. Examination of spinal cord tissue architecture with magnetic resonance diffusion tensor imaging. Neurotherapeutics 2007; 4: 453-459.

Supplementary Information accompanies the paper on the Spinal Cord website (http://www.nature.com/sc) 